\title{
Conventional and reversed partial reinforcement effects in selective learning'
}

\author{
William B. Pavlik, RUTGERS UNIVERSITY \\ William F. Reynolds, QUEENS COLLEGE
}

\begin{abstract}
Abstraet
A reversed PRE was obtained for choice behavior following T-maze training in which CRF and PRSs were "yoked" for correct and incorrect responses. Conventional acquisition procedures yielded differences in acquisition performance and a conventional PRE during extinction. Speed data yielded conventional PREs during extinction in all cases.
\end{abstract}

\section{Problem}

Studies of partial reinforcement extinction effects (PREs) in selective learning situations typically have failed to insure equivalence of behavior of CRF and PR groups during acquisition. A typical finding is that, while PR Ss make more correct responses during extinction than do CRF Ss, CRF Ss typically make more correct responses during acquisition (see Brunswik, 1939; Cotton et al., 1959). Recently, Pavlik \& Born (1962) reported that following a procedure which insured that CRF and PR Ss made equal numbers of correct and incorrect responses during acquisition, the CRF group showed greater resistance to extinction of correct responses than did the PR group. This finding suggests that the conventional PRE observed in selective learning situations, rather than being a direct effect of the schedules, may result from differences between the CRF and PR groups in numbers of correct and incorrect responses made during acquisition. The present study sheds further light on this possible confounding by comparing the effects upon the PRE of acquisition procedures which either permitted CRF and PR Ss to perform differently during acquisition or which required CRF and PR Ss to make the same numbers of correct and incorrect responses during acquisition.

\section{Method}

The apparatus was a single unit $\mathrm{T}$ maze, consisting of a 10-in start box, 24-in stem, 18-in arms, and 12-in goal boxes. Plexiglas doors at the choice point were used to prevent entry into or retracing from an arm of the maze. Photoelectric circuits provided measures of starting time, running speed in the stem, and running speed in each of the arms.

Forty naive male Sprague-Dawley rats, 120-150 days old, were randomly divided into four groups. Group 100 and Group 50 received all freetrials during acquisition. Group $100 \mathrm{Ss}$ were reinforced for each correct response, and Group $50 \mathrm{Ss}$ for an irregular 50\% of correct responses. Group $100 \mathrm{X}$ and $50 \mathrm{X}$ were yoked to Group 50 during acquisition. Each $\mathrm{S}$ in Groups $100 \mathrm{X}$ and $50 \mathrm{X}$ was matched to a $\mathrm{S}$ in Group 50 for number of correct and incorrect responses during each block of 10 acquisition trials. Matching was accomplished by means of forced trials (one door at the choice point was closed). In all cases, as few forced trials were given as was necessary to accomplish the match. Thus, Groups 50,100X, and $50 \mathrm{X}$ made the same numbers of correct and incorrect responses during acquisition. Group 100X Ss were reinforced for each correct response; Group 50X Ss were reinforced for correct responses on the same schedule as obtained for Group 50. Following acquisition, each $\mathrm{S}$ received 30 extinction trials, none of which was forced or reinforced.

A total of 60 acquisition trials were given, 30/day for 2 days. The 30 extinction trials were given on the following day. Noncorrection procedures were used during both acquisition and extinction. All Ss were run under 22-hrs. food deprivation; ad lib water was available in the home cages and in the intertrial holding cage. Each reinforcement consisted of three 45-mg Noyes pellets. Incorrect runs during acquisition and all runs during extinction terminated in $5-\mathrm{sec}$. detention in the empty goal box.

\section{Results}

The choice data are presented in Fig. 1. While Group 100 tended to give more correct responses during acquisition than did Group 50, the difference did not attain significance, $F(1,18)=2.29$. As is apparent in Fig. 1, extinction choice data yield a conventional PRE for Groups 100 and 50, but a reversed PRE for Groups 100X and $50 \mathrm{X}$. This interaction term yielded an $\mathrm{F}(1,36)$ of

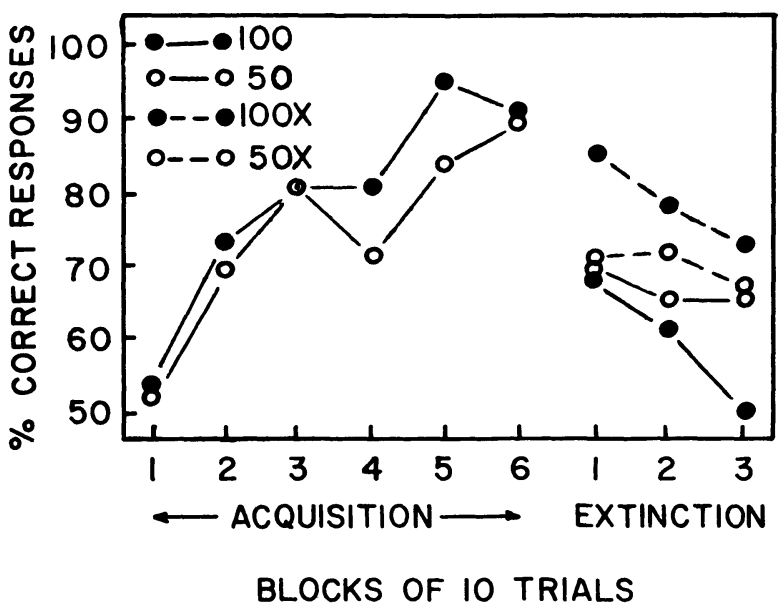

Fig. 1. Mean per cent positive responses during acquisition and extinction. Acquisition curves for Groups $100 \mathrm{X}$ and $50 \mathrm{X}$ would be identical with that of Group 50 . 
5.44, $\mathrm{P}<.05$. Analysis of the interaction by Duncan's M-R Test indicates that Group 100X made significantly more correct responses during extinction than any other group, Group 100 made significantly fewer correct responses than any other group, and that Groups 50 and $50 \mathrm{X}$ did not differ.

All latency data were converted to speed scores (1/sec.). The four speed measures taken during acquisition yielded but one significant between-groups difference; both PR groups ran faster than either CRF group in the Incorrect Arm on Day 2 of acquisition. During extinction, all four speed measures yielded significant effects of Schedules ( $P<.05$ in each case), with PR groups running faster than CRF groups, and significant effects of Trials ( $P<.01$ for each measure), with speed declining across trials. Groups 50 and $50 \mathrm{X}$ did not differ on any speed measure. As the different speed measures yielded generally similar results, only Correct Arm Speed is presented in Fig. 2.

\section{Discussion}

The principal findings of this study are that the equating of PR and CRF groups during acquisition for numbers of correct and incorrect responses by means of a forcing procedure reverses the conventional PRE for choice behavior but has no effect upon the conventional PRE for running speed. Further, the absence of any differences between Groups 50 and 50X, which differed only with respect to the forced trials given Group 50X, suggests that the forcing variable, by itself, is not responsible for these effects.

The acquisition procedures used with Groups $100 \mathrm{X}$ and 50X, which showed a reversed PRE for choice behavior, insured that these groups were equated during acquisition for number of nonreinforced incorrect responses and for number of correct responses, but not for number of reinforced correct responses. If we assume that the equation of incorrect responses during acquisition resulted in equal tendencies for these groups to make incorrect responses during extinction, then the reversed PRE suggests that resistance to extinction of the correct response is a positive function of number of previously

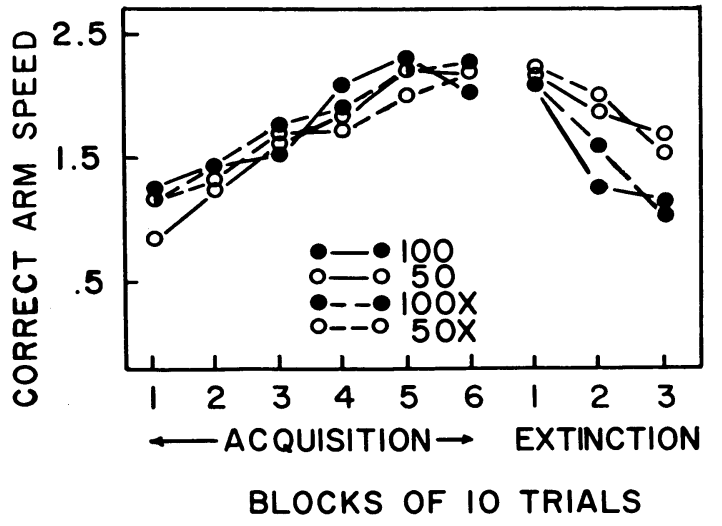

Fig. 2. Mean running speed (ft/sec.) in the correct arm of the maze across blocks of ten trials. reinforced correct responses. With more conventional acquisition procedures, Group 100 made both more correct responses and more reinforced correct responses than didGroup 50. Yet, Group 100 showed less resistance to extinction of correct responses than did Group 50. However, Group 100 also made fewer nonreinforced incorrect responses during acquisition than did Group 50 , and, by assumption, thus had a stronger (less completely habituated) tendency to make incorrect responses during extinction, producing an apparently decreased resistance to extinction of correct response performance. Thus the findings of this study for choice behavior, coupled with the assumption that the tendency to make competing (incorrect) responses during extinction decreases as nonreinforced competing responses during acquisition increase, appears to implicate differential habituation of competing responses as the factor that mediates the conventional relationship between acquisition reinforcement schedules and subsequent resistance to extinction.

The failure to obtain a reversed PRE for Groups $100 \mathrm{X}$ and $50 \mathrm{X}$ with running speed data may be taken to suggest either that these two criterion measures (choice and speed) obey different laws or (perhaps more likely) that they are differentially susceptible to different competing responses. These groups were equated during acquisition for incorrect turns, which should affect the tendency to make correct turns but which may have little if any effect upon running speed. Responses such as stopping and grooming, which may have greater effects upon running speed than upon choice behavior, were not controlled in the present study, though they are known to occur more frequently during acquisition in PR groups than in CRF groups (Goodrich, 1959). If such responses could be equated in PR and CRF groups, then the above reasoning suggests that running also should be more resistant to extinction following CRF than PR acquisition schedules. In any case, the results of the present study suggest that an understanding of the PRE is complicated by the confounding of acquisition reinforcement schedules with acquisition performance differences.

\section{Referenees}

BRUNSWIK, E. Probability as a determiner of rat behavior. J. exp. Psychol., 1939, 25, 175-197.

COTTON, J. W., LEWIS, D. J., \& JENSEN, G. D. Partial reinforcement effects in a T-maze. J.comp. physiol. Psychol., 1959, 52, 730-733.

GOODRICH, K. P. Performance in different segments of an instrumental response chain as a function of reinforcement schedule. J. exp. Psychol., 1959, 57, 57-63. PAVLIK, W. B., \& BORN, D. G. Partial reinforcement effects in selective learning. Psychol. Rep., 1962, 11, 575-580.

\section{Note}

1. This research was supported by funds from USPHS Grant M-6534(A) and from the Rutgers University Research Council. 